\title{
An Output Integral Sliding Mode FTC Scheme Using Control Allocation
}

\author{
Mirza Tariq Hamayun, Halim Alwi and Christopher Edwards
}

\begin{abstract}
In this paper, a new fault tolerant control scheme is proposed for systems where only output information is available. The ideas of integral sliding mode control are used to ensure robustness throughout the entire response of the system, even in certain actuator fault/failure cases. This is accomplished by integrating fixed control allocation within this ISM framework. An unknown input observer is included in the proposed scheme to estimate the states, as it is assumed that there is no information of the true states or knowledge of the faults or failures from a monitoring scheme. A rigorous closed-loop stability analysis is presented, and in fact, a convex representation of the problem is formulated to synthesize a controller. Simulation results on a benchmark civil aircraft model show good tracking of the commanded signals.
\end{abstract}

\section{INTRODUCTION}

Fault tolerant control (FTC) can improve the reliability of safety critical systems. Many different control paradigms have been adopted to tackle the problem, and it remains an open area of research. Overviews of the development of FTC schemes have been provided in the recent book chapter [21] and the references therein.

Control allocation (CA) is one approach which has the capability to effectively manage redundancy in over-actuated systems [4], [13]. The benefit of using CA methods is that they can be used in combination with other control design paradigms to distribute the virtual control effort among the actuators. For a detailed discussion of the advantages of using CA in terms of FTC, see for example [5], [9].

Due to its inherent robustness properties against matched uncertainties, sliding mode control (SMC) [10], [19] has recently attracted much attention in the field of FTC. The combination of traditional SMC with CA for fault tolerant control has been previously explored in [1], [16] and [14]. In all this work it was assumed that the system states are known and that state feedback control schemes could be employed. More recently a FTC approach has been proposed in [12], which considers integral sliding mode (ISM) ideas to achieve fault tolerance. The idea of ISM control originally proposed in [20], [7] is to eliminate the reaching phase associated with SMC schemes.

Early work on ISM assumed state information, but this has been extended to the situation where only measured outputs are available [3]. In [8] the state dependent method to design the integral sliding surface from [6], was developed into an output feedback framework. The main contribution of this paper is to relax the assumption associated with [1], [12] that state information is known, and to consider instead the situation where only measured outputs are available. The proposed scheme includes a full order linear unknown input observer UIO, to estimate the system states used in the design of the (virtual) controller. In the proposed FTC

M.T. Hamayun, H. Alwi and C. Edwards are with the Control Research Group, University of Leicester UK mth11, ha18, ce14@le.ac.uk

The first author acknowledges the support of COMSATS for this research. scheme, both actuator and component faults are considered. As a consequence, the approach is different from the output dependent ISM schemes proposed in [3]. It also allows the presence of invariant zeros, which must be precluded in the approach of [3]. In the scheme no attempt is made to estimate the faults/failures (using an FDI scheme), instead the robustness properties of the UIO coupled with the ISM are relied upon. Although the approach here is similar in spirit to [8] the technical details are different and the control allocation context is new. A rigorous closed-loop stability analysis is carried out to ensure the stability of the sliding motion in the face of faults/failures, provided that redundancy is available in the system.

\section{Problem Formulation}

Consider an uncertain system with actuator faults or failures and component faults written as

$$
\begin{aligned}
\dot{x}(t) & =\left(A+A^{\delta}\right) x(t)+B u(t)-B K u(t) \\
y(t) & =C x(t)
\end{aligned}
$$

where $A \in \mathbb{R}^{n \times n}$ is the state matrix, $A^{\delta}$ is parametric uncertainty in the system matrix arising from faults at a component level, $B \in \mathbb{R}^{n \times m}$ is the input distribution matrix and $C \in \mathbb{R}^{p \times n}$ is the output distribution matrix. The diagonal weighting matrix $K=\operatorname{diag}\left\{k_{1}, . ., k_{m}\right\}$, where the scalars $k_{1}, . ., k_{m}$, models the effectiveness level of the actuators. If $k_{i}=0$, the corresponding $i$ th actuator is fault free and is working perfectly, whereas if $1>k_{i}>0$, an actuator fault is present. The value $k_{i}=1$ indicates the $i$ th actuator has completely failed. It is assumed the outputs to be controlled are given by $y_{c}(t)=C_{c} x(t)$ where $C_{c} \in \mathbb{R}^{l \times n}$, where $l<m$. It follows that there is redundancy in the system in terms of the number of control inputs. This will be exploited to achieve fault tolerance. To resolve this redundancy, as in [1], it is assumed the matrix $B$ can be partitioned such that

$$
B=\left[\begin{array}{ll}
B_{1}^{T} & B_{2}^{T}
\end{array}\right]^{T}
$$

where $B_{1} \in \mathbb{R}^{(n-l) \times m}$ and $B_{2} \in \mathbb{R}^{l \times m}$ is of rank $l<m$. By appropriate scaling of the last $l$ states, it can be ensured $B_{2} B_{2}^{T}=I_{l}$, which implies $\left\|B_{2}\right\|=1$. As in [1], it is further assumed that $\left\|B_{1}\right\| \ll\left\|B_{2}\right\|=1$, so that $B_{2}$ reflects that the dominant control action contribution on the system acts in the lower $l$ channels of the system. As argued in [1], such a partition can be achieved for aerospace systems for example. Using (2), the system in (1) can be written as

$$
\dot{x}(t)=\left(A+A^{\delta}\right) x(t)+\left[\begin{array}{c}
B_{1} \\
B_{2}
\end{array}\right] \underbrace{(I-K)}_{W} u(t)
$$

Notice, by definition $W:=I-K$ is a diagonal matrix and its diagonal elements $w_{i}$ satisfy $0 \leq w_{i} \leq 1$. 
The physical control law $u(t)$ is realized by a so-called fixed control allocation scheme of the form

$$
u(t)=B_{2}^{T} v(t)
$$

where $v(t) \in \mathbb{R}^{l}$ is the virtual control input. (In (4) the fact that $B_{2} B_{2}^{\mathrm{T}}=I_{l}$ is exploited). Using (4), equation (3) can be written as

$$
\dot{x}(t)=\left(A+A^{\delta}\right) x(t)+\underbrace{\left[\begin{array}{c}
B_{1} W B_{2}^{T} \\
B_{2} W B_{2}^{T}
\end{array}\right]}_{B_{w}} v(t)
$$

In the nominal case, when there is no fault $W=I$ and $A^{\delta}=0$, using the fact that $B_{2} B_{2}^{T}=I_{l}$, equation (5) simplifies to

$$
\dot{x}(t)=A x(t)+\underbrace{\left[\begin{array}{c}
B_{1} B_{2}^{T} \\
I_{l}
\end{array}\right]}_{B_{v}} v(t)
$$

The following assumption will be made:

A1: $\quad$ The pair $\left(A, B_{v}\right)$ is controllable.

The design of the virtual control law $v(t)$ will be based on an estimate of the states $\hat{x}(t)$, rather than the true states $x(t)$.

\section{ISM CONTROLLER DESIGN}

In this section, a step by step design procedure for the synthesis of the virtual control $v(t)$ will be developed using the estimated states $\hat{x}(t)$, which will be obtained from an observer. As a first design step, an output and state estimate dependent integral switching function is proposed of the form

$$
\sigma(t)=G y(t)-G y\left(t_{0}\right)+\int_{0}^{t} F \hat{x}(\tau) d \tau
$$

where $G \in \mathbb{R}^{l \times p}$ is design freedom, and $F \in \mathbb{R}^{l \times n}$ is a feedback gain, which is to be designed to achieve appropriate nominal closed-loop performance. The formulation in (7) is similar to that proposed in [8] except here both $F$ and $G$ depend on the dimension $l$ rather than the number of control inputs. The second design step is the creation of a control law $v(t)$ to ensure the switching function is zero for all time. In this paper, the full-order UIO developed in [15] is used to estimate the system states. The term $B K u(t)$ in (1) is regarded as an unknown input since by assumption $K$ is unknown. Necessary and sufficient conditions for a linear UIO to exist to ensure insensitivity to $B K u(t)$ are

A2: $\operatorname{rank}(C B)=\operatorname{rank}(B)=m$

$A 3$ : the triple $(A, B, C)$ is minimum phase

The structure of the full-order observer from [15] is

$$
\begin{aligned}
& \dot{z}(t)=A_{0} z(t)+T B u(t)+L y(t) \\
& \hat{x}(t)=z(t)+H y(t)
\end{aligned}
$$

where $\hat{x}(t)$ is the estimated state, and $A_{0}, T, L$ and $H$ are matrices of appropriate dimension (and include design parameters). From [15], the matrix $H \in \mathbb{R}^{n \times p}$ is chosen so that

$$
(I-H C) B=0
$$

As argued in [15], Assumption $A 2$ is sufficient to solve (10) and $H=B\left((C B)^{T} C B\right)^{-1}(C B)^{T}$ is an appropriate choice. Once $H$ is computed, the matrices

$$
\begin{aligned}
T & :=I-H C \\
A_{0} & :=\underbrace{A-H C A}_{A_{h}}-L_{1} C
\end{aligned}
$$

can be defined, where $L_{1} \in \mathbb{R}^{n \times p}$ is design freedom chosen to make $A_{0}$ Hurwitz. Finally

$$
L_{2}:=A_{0} H
$$

and the gain $L:=L_{1}+L_{2}$

\section{Remarks:}

- The approach in this paper can tolerate the presence of stable invariant zeros associated with the triple $(A, B, C)$ as indicated in assumption $A 3$. This precludes the use of the strong stability approach in [3].

- In the original paper [15], the stated conditions for solving (10)-(13) are that $\operatorname{rank}(C B)=\operatorname{rank}(B)$ and the pair $\left(C, A_{1}\right)$ is detectable where $A_{1}=A-$ $B\left((C B)^{T} C B\right)^{-1}(C B)^{T} C A$. It is argued in [18] these are equivalent to $A 2$ and $A 3$.

If $e(t)=x(t)-\hat{x}(t)$, using the plant equation in (1), after some algebra and simplifications based on (10)-(13), the error dynamics can be written as

$$
\begin{aligned}
\dot{e}(t) & =A_{0} e(t)+T A^{\delta} e(t)+T A^{\delta}(x(t)-e(t)) \\
& =A_{0} e(t)+T A^{\delta} x(t)
\end{aligned}
$$

The choice of $G$ in (7) suggested in this paper is

$$
G:=B_{2}\left((C B)^{T} C B\right)^{-1}(C B)^{T}
$$

where the existence of the inverse is guaranteed by assumption $A 2$. This choice of $G$ is different to the suggested form in [8], and the non-square term $B_{2}$ must be included here to account for the fact that $C B \in \mathbb{R}^{p \times m}$, where $m>l$. Suppose a control law can be designed to force $\sigma=\dot{\sigma}=0$ for all time. The equivalent control signal $v_{e q}(t)$ necessary to maintain sliding is obtained from the equation $\dot{\sigma}=0$ [10]. Taking the time derivative of equation (7) yields

$$
\dot{\sigma}(t)=G \dot{y}(t)+F \hat{x}(t)
$$

and substituting from equation (5) into the above, and equating $\dot{\sigma}(t)=0$, results in the expression

$$
v_{e q}(t)=-\left(G C B_{w}\right)^{-1}\left(F \hat{x}(t)+G C\left(A+A^{\delta}\right) x(t)\right)
$$

under the assumption $\operatorname{det}\left(G C B_{w}\right) \neq 0$. With the choice of $G$ in (15), $G C B_{w}=B_{2} W B_{2}^{T}$ and with the assumption that $e(t)=x(t)-\hat{x}(t)$, the expression in (17) becomes

$$
v_{e q}(t)=-\left(B_{2} W B_{2}^{T}\right)^{-1}\left(F x(t)-F e(t)+G C\left(A+A^{\delta}\right) x(t)\right)
$$

Substituting (18) into (5) to obtain the sliding dynamics yields the expression

$$
\dot{x}(t)=\left(A+A^{\delta}\right) x(t)-B_{m}\left(F x(t)-F e(t)+G C\left(A+A^{\delta}\right) x(t)\right)
$$

where the matrix

$$
B_{m}:=\left[\begin{array}{c}
B_{1} W B_{2}^{T}\left(B_{2} W B_{2}^{T}\right)^{-1} \\
I_{l}
\end{array}\right]
$$


Define two matrices $\widetilde{B}_{1}=\left[\begin{array}{ll}I_{n-l} & 0\end{array}\right]^{T}$ and $\widetilde{B}_{2}=\left[\begin{array}{ll}0 & I_{l}\end{array}\right]^{T}$ so that $\left[\begin{array}{cc}\widetilde{B}_{1} & \widetilde{B}_{2}\end{array}\right]=I_{n}$. Since

$$
B_{m}=\underbrace{\left[\begin{array}{c}
0 \\
I_{l}
\end{array}\right]}_{\widetilde{B}_{2}}+\underbrace{\left[\begin{array}{c}
I_{n-l} \\
0
\end{array}\right]}_{\widetilde{B}_{1}} \underbrace{B_{1} W B_{2}^{T}\left(B_{2} W B_{2}^{T}\right)^{-1}}_{\psi(t)}
$$

the sliding dynamics in (19), can be written as

$$
\begin{aligned}
\dot{x}(t)= & \left(A-\widetilde{B}_{2} F+A^{\delta}\right) x(t)-\widetilde{B}_{1} \psi(t) F x(t)+\widetilde{B}_{2} F e(t) \\
& +\widetilde{B}_{1} \psi(t) F e(t)-\widetilde{B}_{2} G C A x(t)-\widetilde{B}_{2} G C A^{\delta} x(t) \\
& -\widetilde{B}_{1} \psi(t) G C A x(t)-\widetilde{B}_{1} \psi(t) G C A^{\delta} x(t) \\
= & \left(A-\widetilde{B}_{2} F-\widetilde{B}_{2} G C A\right) x(t)-\widetilde{B}_{1} \psi(t) F x(t) \\
& -\widetilde{B}_{1}\left(\psi(t) G C A x(t)+\psi(t) G C A^{\delta} x(t)\right) \\
& +\left(I-\widetilde{B}_{2} G C\right) A^{\delta} x(t)+\widetilde{B}_{1} \psi(t) F e(t)+\widetilde{B}_{2} F e(t)
\end{aligned}
$$

Combining equations (14) and (21), the closed-loop system dynamics can be written as

$$
\left[\begin{array}{l}
\dot{e}(t) \\
\dot{x}(t)
\end{array}\right]=\underbrace{\left[\begin{array}{cc}
A_{0} & 0 \\
\widetilde{B}_{2} F & A_{c}-\widetilde{B}_{2} F
\end{array}\right]}_{A_{a}} \underbrace{\left[\begin{array}{l}
e(t) \\
x(t)
\end{array}\right]}_{x_{a}}+B_{a} \Delta C_{a}\left[\begin{array}{l}
e(t) \\
x(t)
\end{array}\right]
$$

where

$$
\begin{aligned}
A_{c} & :=\left(I-\widetilde{B}_{2} G C\right) A \\
B_{a} & :=\left[\begin{array}{ccc}
T & 0 & 0 \\
\left(I-\widetilde{B}_{2} G C\right) & -\widetilde{B}_{1} & -\widetilde{B}_{1}
\end{array}\right] \\
C_{a} & :=\left[\begin{array}{cc}
0 & I \\
-F & G C A+F \\
0 & I
\end{array}\right]
\end{aligned}
$$

and the uncertainty

$$
\Delta:=\operatorname{diag}\left[\begin{array}{lll}
A^{\delta} & \psi & \psi G C A^{\delta}
\end{array}\right]
$$

It is convenient to analyze (22) in the $(e, \hat{x})$ coordinates. Since

$$
\underbrace{\left[\begin{array}{l}
e(t) \\
\hat{x}(t)
\end{array}\right]}_{\hat{x}_{a}}=\underbrace{\left[\begin{array}{cc}
I & 0 \\
-I & I
\end{array}\right]}_{\widetilde{T}}\left[\begin{array}{l}
e(t) \\
x(t)
\end{array}\right]
$$

it follows

$$
\begin{aligned}
& \widetilde{A}_{a}:=\widetilde{T} A_{a} \widetilde{T}^{-1}=\left[\begin{array}{cc}
A_{0} & 0 \\
A_{c}-A_{0} & A_{c}-\widetilde{B}_{2} F
\end{array}\right] \\
& \widetilde{B}_{a}:=\widetilde{T} B_{a}=\left[\begin{array}{ccc}
T & 0 & 0 \\
\left(I-\widetilde{B}_{2} G C\right)-T & -\widetilde{B}_{1} & -\widetilde{B}_{1}
\end{array}\right] \\
& \widetilde{C}_{a}:=C_{a} \widetilde{T}^{-1}=\left[\begin{array}{cc}
I & I \\
G C A & G C A+F \\
I & I
\end{array}\right]
\end{aligned}
$$

and in the $(e, \hat{x})$ coordinates, equation (22) can be written as

$$
\dot{\hat{x}}_{a}(t)=\widetilde{A}_{a} \hat{x}_{a}(t)+\widetilde{B}_{a} \Delta \widetilde{C}_{a} \hat{x}_{a}(t)
$$

Now in order to determine that the term $\psi(t)$ in (20) is bounded, note that $\psi(t)=B_{1} B_{2}^{\dagger}(t)$, where $B_{2}^{\dagger}(t)$ is a right pseudo inverse of $B_{2}$. Using the pseudo inverse properties in [17], and arguing as in [1], there exists a scalar $\gamma_{0}$ such that

$$
\left\|B_{2}^{\dagger}(t)\right\|:=\left\|W B_{2}^{T}\left(B_{2} W B_{2}^{T}\right)^{-1}\right\|<\gamma_{0}
$$

for all combinations of $0<w_{i} \leq 1$. Therefore

$$
\|\psi(t)\| \leq \gamma_{1} \gamma_{0}
$$

where $\gamma_{1}=\left\|B_{1}\right\|$, which is assumed to be small.

A4: $\quad$ It is assumed that the parametric uncertainty $A^{\delta}$ in the system matrix $A$ is bounded so that

$$
\|\Delta\|<\gamma_{a}
$$

\section{A. Closed-loop Stability Analysis}

In the nominal case, i.e. when $K=0$, and $\Delta=0$, equation (31) is stable by design. However for the fault/failure cases, stability needs to be established. To this end define

$$
\gamma_{2}=\left\|\widetilde{G}_{a}(s)\right\|_{\infty}
$$

where

$$
\widetilde{G}_{a}(s):=\widetilde{C}_{a}\left(s I-\widetilde{A}_{a}\right)^{-1} \widetilde{B}_{a}
$$

Proposition 1: During fault or failure conditions, for any $0 \leq k_{i}<1$, the closed loop system will be stable if:

$$
\gamma_{2} \gamma_{a}<1
$$

Proof: The closed-loop system defined in (31), can also be written as

$$
\begin{aligned}
& \dot{\hat{x}}_{a}(t)=\widetilde{A}_{a} \hat{x}_{a}(t)+\widetilde{B}_{a} \widetilde{u}_{a}(t) \\
& \widetilde{y}_{a}(t)=\widetilde{C}_{a} \hat{x}_{a}(t)
\end{aligned}
$$

where

$$
\widetilde{u}_{a}(t)=\Delta \widetilde{y}_{a}(t)
$$

In this form, equation (31), can be considered as the feedback interconnection of the known linear system $\widetilde{G}_{a}(s)$, and the bounded uncertain system $\Delta$. According to the small gain theorem, the feedback interconnection of $\widetilde{G}_{a}(s)$ and $\Delta$, and hence equation (31), will be stable if

$$
\gamma_{2} \gamma_{a}<1
$$

and the proof of this proposition is complete.

\section{B. LMI Synthesis}

This section considers the synthesis of the design gain $L_{1}$ and $F$ so that (37) is satisfied. For the triple $\left(\widetilde{A}_{a}, \widetilde{B}_{a}, \widetilde{C}_{a}\right)$ the Bounded Real Lemma (BRL) can be expressed in the form: $\left\|\widetilde{G}_{a}(s)\right\|_{\infty}<\gamma_{2}$ where

$$
\widetilde{G}_{a}(s):=\widetilde{C}_{a}\left(s I-\widetilde{A}_{a}\right)^{-1} \widetilde{B}_{a}
$$

iff there exists a s.p.d matrix $X \in \mathbb{R}^{2 n \times 2 n}$ such that

$$
\left[\begin{array}{ccc}
\widetilde{A}_{a} X+X \widetilde{A}_{a}^{\mathrm{T}} & \widetilde{B}_{a} & X \widetilde{C}_{a}^{\mathrm{T}} \\
\widetilde{B}_{a}^{T} & -\gamma_{2}^{2} I & 0 \\
\widetilde{C}_{a} X & 0 & -I
\end{array}\right]<0
$$

Here it is assumed that $X=\operatorname{diag}\left(X_{1}, X_{2}\right)$ where the two subblocks $X_{1}, X_{2} \in \mathbb{R}^{n \times n}$ are s.p.d. As a result of this assumption

$$
\widetilde{C}_{a} X=\left[\begin{array}{cc}
X_{1} & X_{2} \\
G C A X_{1} & G C A X_{2}+Y \\
X_{1} & X_{2}
\end{array}\right]
$$


where $Y:=F X_{2}$. The top left subblock in (43)

$$
\widetilde{A}_{a} X+X \widetilde{A}_{a}^{\mathrm{T}}=\left[\begin{array}{cc}
A_{0} X_{1}+X_{1} A_{0}^{\mathrm{T}} & X_{1} A_{c}^{\mathrm{T}}-X_{1} A_{0}^{\mathrm{T}} \\
A_{c} X_{1}-A_{0} X_{1} & \Theta
\end{array}\right]
$$

where $\Theta=A_{c} X_{2}+X_{2} A_{c}^{\mathrm{T}}-\widetilde{B}_{2} Y-Y^{\mathrm{T}} \widetilde{B}_{2}^{T}$. Also write $A_{0}=A_{h}-$ $L_{1} C$ where $A_{h}$ is from (12). To create a convex representation, define the observer gain

$$
L_{1}:=\beta B E
$$

where $\beta$ is a positive scalar and $E \in \mathbb{R}^{m \times p}$ is chosen so that $\left(A_{h}, B, E C\right)$ is minimum phase. This is possible if $(A, B, C)$ is minimum phase [11]. Then as argued in [11] it is possible to find an s.p.d matrix $P$ which has a structure $P=N^{\mathrm{T}} \operatorname{diag}\left(P_{1}, P_{2}\right) N$ such that $P B=(E C)^{\mathrm{T}}$, where $N \in \mathbb{R}^{n \times n}$ is invertible and depends on $E$ and the s.p.d. matrices $P_{1} \in \mathbb{R}^{(n-m) \times(n-m)}, P_{2} \in \mathbb{R}^{m \times m}$. The matrix $N$ is in fact associated with a linear change of coordinates to force the triple $\left(A_{h}, B, E C\right)$ into the canonical form proposed in [11]. Define $X_{11}=P_{1}^{-1}$ and $X_{12}=P_{2}^{-1}$. It follows that $L_{1} C=\beta B E C=\beta B B^{\mathrm{T}} P$ and so if

$$
X_{1}:=P^{-1}=N^{-1} \operatorname{diag}\left(X_{11}, X_{12}\right)\left(N^{-1}\right)^{\mathrm{T}}>0
$$

then $L_{1} C X_{1}=\beta B B^{\mathrm{T}}$ and $A_{0} X_{1}=A_{h} X_{1}-\beta B B^{\mathrm{T}}$. It follows the $\mathrm{BRL}$ in (43) is affine with respect to the decision variables $X_{11}, X_{12}, X_{2}, \beta, Y$ and so the synthesis problem is convex.

For the nominal system in (6), when $W=I$ and $A^{\delta}=0$, the matrix $F$ must be chosen to stabilize $\left(A-B_{V} F\right)$. Since $\left(A, B_{V}\right)$ is assumed to be controllable, an LQR formulation will be adopted where $F$ is chosen to minimize

$$
J=\int_{0}^{\infty}\left(x^{T} Q x+v^{T} R v\right) d t
$$

where $Q$ and $R$ are symmetric positive definite design matrices. This problem can be posed as an LMI optimization Minimize trace $\left(X_{2}^{-1}\right)$ subject to

$$
\left[\begin{array}{cc}
A X_{2}+X_{2} A^{T}-B_{v} Y-Y^{T} B_{v}^{T} & \left(Q X_{2}-R Y\right)^{T} \\
Q X_{2}-R Y & -I
\end{array}\right]<0
$$

For a given $\mathscr{L}_{2}$-gain $\gamma_{2}$, the overall optimization problem proposed in convex form becomes:

Minimize $\operatorname{trace}(Z)$ with respect to the decision variables $X_{11}, X_{12}, X_{2}, \beta, Y$ subject to

$$
\left[\begin{array}{cc}
-Z & I_{n} \\
I_{n} & -X_{2}
\end{array}\right]<0
$$

together with (43), (48), (47) and 49. The matrix $Z$ is a slack variable which satisfies $Z>X_{2}^{-1}$ and therefore $\operatorname{trace}(Z) \geq$ trace $\left(X_{2}^{-1}\right)$. Finally the gain can be recovered as $F=Y X_{2}^{-1}$.

\section{ISM Control Laws}

The integral sliding mode control law, which is based on the nominal system (6) and applied in (6) is defined as

$$
v(t)=v_{l}(t)+v_{n}(t)
$$

where the linear part, which is responsible for the nominal performance of the system is given by

$$
v_{l}(t)=-F \hat{x}(t)-G C A \hat{x}(t)
$$

and the nonlinear part, which induces sliding is defined as

$$
v_{n}(t)=-\rho \frac{\sigma(t)}{\|\sigma(t)\|} \quad \text { for } \sigma(t) \neq 0
$$

where $\rho$ is a suitable scalar gain.

Now in the sequel, it is demonstrated that the control law defined in (50) satisfies the standard reachability condition [10]. To demonstrate this, by using the relation $K=I-W$, equation (16) can be written as

$$
\begin{aligned}
\dot{\sigma}(t) & =G C\left(A+A^{\delta}\right) x(t)+\left(B_{2} W B_{2}^{T}\right) v(t)+F \hat{x}(t) \\
& =G C\left(A+A^{\delta}\right) x(t)+v(t)-\left(I-B_{2} W B_{2}^{T}\right) v(t)+F \hat{x}(t) \\
& =G C\left(A+A^{\delta}\right) x(t)+v(t)-\left(B_{2}(I-W) B_{2}^{T}\right) v(t)+F \hat{x}(t) \\
& =G C\left(A+A^{\delta}\right) x(t)+v(t)-B_{2} K B_{2}^{T} v(t)+F \hat{x}(t)
\end{aligned}
$$

Substituting the control law (50)-(52), into the expression above and exploiting the fact that $e(t)=x(t)-\hat{x}(t)$ yields

$$
\begin{aligned}
\dot{\sigma}(t)= & G C A^{\delta} \hat{x}(t)+G C A^{\delta} e(t)+G C A e(t) \\
& -\left(B_{2} K B_{2}^{T}\right)\left(v_{l}+v_{n}\right)-\rho \frac{\sigma(t)}{\|\sigma(t)\|}
\end{aligned}
$$

Proposition 2: The control law defined in (50), ensures that the system trajectories remain on the sliding surface, if the modulation gain $\rho(t)$ is defined as

$$
\rho(t)>\frac{\left\|G C A^{\delta}\right\|\|\hat{x}\|+\left\|v_{l}\right\|+\left(\|G C A\|+\left\|G C A^{\delta}\right\|\right)\|e\|+\eta}{\left(1-\lambda_{0}\right)}
$$

where $\eta$ is a positive design scalar, and the fault associated with the tuple $\left(k_{1}, \ldots, k_{m}\right)$ is assumed to belong to a set $\mathscr{D}=$ $\left\{\left(k_{1}, \ldots, k_{m}\right): \lambda_{\text {max }}\left(B_{2} K B_{2}^{T}\right)<\lambda_{0}<1\right\}$.

Proof: Taking the time derivative of the candidate Lyapunov function $V=0.5 \sigma^{T} \sigma$ and substituting from (54) yields

$$
\begin{aligned}
\dot{V}= & \sigma^{T}\left(G C A^{\delta} \hat{x}(t)+G C A^{\delta} e(t)+G C A e(t)\right. \\
& \left.-\left(B_{2} K B_{2}^{T}\right)\left(v_{l}+v_{n}\right)-\rho \frac{\sigma(t)}{\|\sigma(t)\|}\right) \\
\leq & \|\sigma\|\left(\left\|G C A^{\delta}\right\|\|\hat{x}\|+\left(\left\|G C A^{\delta}\right\|+\|G C A\|\right)\|e\|\right. \\
& \left.+\left\|B_{2} K B_{2}^{T}\right\|\left\|v_{l}\right\|-\rho\left(1-\left\|B_{2} K B_{2}^{T}\right\|\right)\right)
\end{aligned}
$$

Since $\left\|B_{2} K B_{2}^{T}\right\| \leq 1$, for a fault represented by $\left(k_{1}, \ldots k_{m}\right) \in$ $\mathscr{D}$, if the expression for $\rho(t)$ in (55) holds, the inequality (56) can be written as

$$
\dot{V} \leq-\eta\|\sigma\|=-\eta \sqrt{2 V}
$$

which is a standard reachability condition. This guarantees that a sliding motion is maintained for all subsequent time.

Finally the physical control law $u(t)$ is obtained by substituting equations (50)-(52) into (4) to obtain

$$
u(t)=B_{2}^{T}\left(-F \hat{x}(t)-G C A \hat{x}(t)-\rho \frac{\sigma(t)}{\|\sigma(t)\|}\right)
$$

In order to get an expression for the upper bound of the unknown signal $e(t)$ in (54), equation (14) can be written as

$$
\dot{e}(t)=\left(A_{0}+T A^{\delta}\right) e(t)+T A^{\delta} \hat{x}(t)
$$


Suppose $\left\|A^{\delta}\right\|$ is sufficiently small that for the Lyapunov matrix $P_{0}$ satisfying $P_{0} A_{0}+A_{0}^{T} P_{0}=-I$, the uncertainty satisfies

$$
2\left\|P_{0}\right\|\left\|T A^{\delta}\right\|<1-\mu_{o} \quad \text { where } \quad \mu_{o}>0
$$

Then for $V_{0}=e^{T} P_{0} e$

$$
\begin{aligned}
\dot{V}_{0} & =e\left(P_{0} A_{0}+A_{0}^{T} P_{0}\right) e^{T}+2 e^{T} P_{0} T A^{\delta} e+2 e^{T} P_{0} T A^{\delta} \hat{x} \\
& \leq-\|e\|^{2}+2\|e\|^{2}\left\|P_{0}\right\|\left\|T A^{\delta}\right\|+2\|e\|\left\|P_{0}\right\|\left\|T A^{\delta}\right\|\|\hat{x}\|
\end{aligned}
$$

and therefore

$$
\begin{aligned}
\dot{V}_{0} & \leq-\mu_{0}\|e\|^{2}+\left(1-\mu_{0}\right)\|\hat{x}\|\|e\| \\
& \leq-\frac{\mu_{0}}{\lambda_{\max }\left(P_{0}\right)} V_{0}+\frac{1-\mu_{0}}{\sqrt{\lambda_{\max }\left(P_{0}\right)}}\|\hat{x}\| \sqrt{V_{0}}
\end{aligned}
$$

Define $\widetilde{V}=\sqrt{V_{0}}$, then (61) implies

$$
\dot{\tilde{V}} \leq-\frac{\mu_{0}}{2 \lambda_{\max }\left(P_{0}\right)} \widetilde{V}+\frac{1-\mu_{0}}{2 \sqrt{\lambda_{\max }\left(P_{0}\right)}}\|\hat{x}\|
$$

For notational convenience write this as

$$
\dot{\widetilde{V}} \leq-m_{0} \widetilde{V}+m_{1}\|\hat{x}\|
$$

where $m_{0}$, and $m_{1}$ are appropriately defined positive scalars. Define

$$
\dot{r}(t)=-m_{0} r(t)+m_{1}\|\hat{x}(t)\|
$$

Then if $r(0)>\widetilde{V}(0)$, it can be shown that $r(t)>\widetilde{V}(t)$ for all $t \geq 0$ and consequently

$$
r(t) \geq \sqrt{\lambda_{\max }\left(P_{0}\right)}\|e(t)\| \text { for } t \geq 0
$$

Therefore $r(t)$ can be used as an upper bound on the unknown signal $e(t)$ in the control law. Clearly the filter defined in (64) can be realized in real time, and hence $r(t)$ can be used as an upper bound on the unknown estimation error $e(t)$. Finally the modulation gain associated with the unit vector in the control law in (54) is given by

$$
\rho(t)=\frac{\left\|G C A^{\delta}\right\|\|\hat{x}\|+\left\|v_{l}\right\|+r(t)\left(\|G C A\|+\left\|G C A^{\delta}\right\|\right) /\left\|P_{0}\right\|+\eta}{\left(1-\lambda_{0}\right)}
$$

where $r(t)$ is given by (64).

\section{Simulations}

The civil aircraft benchmark model from [2] will be used in simulation to demonstrate the effectiveness, and fault tolerant nature of the proposed scheme. To design the linear component of the controller in (51), the aircraft model has been linearized around an operating condition of straight and level flight with a mass of $263,000 \mathrm{Kg}, 92.6 \mathrm{~m} / \mathrm{s}$ true airspeed, and at an altitude of $600 \mathrm{~m}$ based on $25.6 \%$ of maximum thrust and at a 20 deg flap position. The linearized state space model is given in [2].

In the simulations, only longitudinal control is considered. The system states are $x(t)=\left(q, V_{t a s}, \alpha, \theta\right)^{T}$ where $q$ is the pitch rate $(\mathrm{rad} / \mathrm{sec}), V_{\text {tas }}$ is the true airspeed $(\mathrm{m} / \mathrm{sec}), \alpha$ is the angle of attack (rad) and $\theta$ is the pitch angle (rad). It is assumed that the measured system outputs available for use in the control law are $y=C_{p} x(t)=\left(q, V_{t a s}, \theta\right)^{T}$. The available control surfaces for the longitudinal control are $\delta_{\text {long }}=\left[\delta_{e}, \delta_{s}, \delta_{e p r}\right]^{T}$ which represent elevator deflection (rad), horizontal stabilizer deflection (rad) and aggregated longitudinal EPR (i.e. the four individual engine pressure ratios (EPRs) aggregated to produce one control input). A separate inner-loop Proportional Integral (PI) controller to held true airspeed $V_{t a s}$ at a constant level is used which uses the error in speed to manipulate EPR. It is assumed the engines are fault free. Rewrite the linear model

$$
\begin{aligned}
\dot{x}(t) & =A_{p} x(t)+B_{s} u_{1}+B_{e} \delta_{e p r} \\
y & =C_{p} x(t)
\end{aligned}
$$

where $u_{1}=\left[\delta_{e}, \delta_{s}\right]^{T}$. The matrices $B_{s} \in \mathbb{R}^{4 \times 2}$ and $B_{e} \in \mathbb{R}^{4 \times 1}$ are the input distribution matrices associated with $\left[\delta_{e}, \delta_{s}\right]^{T}$ and $\delta_{e p r}$ respectively. Define a new state associated with the PI controller as

$$
\dot{x}_{r}=R_{r_{1}}-C_{1} x
$$

where $R_{r_{1}}(t)$ is the reference signal for $V_{\text {tas }}$ tracking and $C_{1}=\left[\begin{array}{cccc}0 & 1 & 0 & 0\end{array}\right]$. The inner loop PI control is given by $\delta_{e p r}=K_{p}\left(R_{r_{1}}(t)-C_{1} x(t)\right)+K_{i} x_{r}(t)$, where the PI gains are chosen as $K_{p}=0.2$, and $K_{i}=0.1$. Augmenting the plant in (67) with $x_{r}(t)$ yields

$$
\left[\begin{array}{c}
\dot{x}_{r} \\
\dot{x}
\end{array}\right]=\underbrace{\left[\begin{array}{cc}
0 & -C_{1} \\
B_{e} K_{i} & \left(A_{p}-B_{e} K_{p} C_{1}\right)
\end{array}\right]}_{A} \underbrace{\left[\begin{array}{c}
x_{r} \\
x
\end{array}\right]}_{x_{a}(t)}+\underbrace{\left[\begin{array}{c}
0 \\
B_{S}
\end{array}\right]}_{B} u_{1}+\underbrace{\left[\begin{array}{c}
I \\
B_{e} K_{p}
\end{array}\right]}_{B_{r}} R_{r_{1}}
$$

The matrix $B$ needs to be partitioned according to (2) and further scaled to ensure that $B_{2} B_{2}^{T}=I_{l}$, where in this example $l=1$. It is assumed that $x_{r}(t)$ is available to the control law for flight path and therefore $y=C x_{a}(t)$ where $C=\operatorname{diag}\left(1, C_{p}\right)$. The fault tolerant control design will now be based on the system in (70) governed by the triple $(A, B, C)$. It can be verified Assumption $A 2$ holds for this example, since $\operatorname{rank}(C B)=\operatorname{rank}(B)=2$. The triple $(A, B, C)$ has one stable invariant zero. The controlled output for the FPA tracking is given by $y_{c}(t)=C_{c} x(t)$, where $C_{c}=\left[\begin{array}{lllll}0 & 0 & 0 & -1 & 1\end{array}\right]$. To introduce potential faults which cause changes to the aerodynamics to the aircraft, a 10\% change in the aerodynamic coefficients (due to airframe damage) is considered in the simulation. For FPA tracking, the linear part of the virtual control $v_{l}(t)$ in (51) is augmented with a feedforward term $L_{r} R_{r_{2}}(t)$ yielding $v_{l}(t)=L_{r} R_{r_{2}}(t)-F \hat{x}(t)-G C A \hat{x}(t)$ where $L_{r}=C_{c}\left(B_{v} F-A\right)^{-1} B_{v}=6.2281$ and $R_{r_{2}}(t)$ is the FPA reference signal. The choice of $G$ using (15) is $G=\left[\begin{array}{llll}0 & 0.6694 & 0 & 0\end{array}\right]$. For the observer gain $L_{1}$ in (46), $E$ has been chosen such that $E C B=I$, and $\left(A_{h}, B, E C\right)$ is minimum phase with stable zeros at $\{-1.0000,-0.6451,-1.0000\}$. Choosing $Q=\operatorname{diag}(0.02,0.5,0.2,0.1,20)$ and $R=1$ from (48) the feedback gain matrix $F$, obtained by solving the associated LMIs is given by $F=\left[\begin{array}{llllll}-0.0467 & 7.3038 & -0.9965 & -0.0371 & 10.9671\end{array}\right]$ In the closed-loop stability analysis the engines thrust is assumed to be fault free. Based on this assumption, using a numerical search, it can be verified using (26) that the value of $\gamma_{a}$ in (34) is $\gamma_{a}=0.2314$. To satisfy the closedloop stability condition in (37), the scalar $\gamma_{2}$ must satisfy $\gamma_{2}<\frac{1}{0.2314}=4.3215$, which is satisfied through the design of $F$ and $L_{1}$ in the proposed LMIs. 


\section{A. Simulation Results}

The effectiveness of the proposed FTC scheme is tested by considering the performance of the linear civil aircraft model, nominally (when there is no fault), and then through some severe failure tests. To smooth the discontinuity due to the nonlinear control term in (52), a sigmoidal approximation of the form $\frac{\sigma}{\|\sigma\|+\delta}$ has been used, where the value of the positive scalar $\delta$ is chosen as $\delta=0.01$. The value of the modulation gain is chosen here as $\rho=1$. In Figure 1, good tracking of the command signals is achieved. The initial conditions for the plant and observer are taken as $x_{0}=[0,0,0,0]^{T}$, and $x_{0_{o b s}}=[0,0,0,0,0.5(\pi / 180)]^{T}$ respectively. Figures 1 shows the system performance, when an elevator jams at some offset position. It can be seen that the proposed scheme makes the horizontal stabilizer more active to keep the performance close to nominal, by maintaining the sliding motion. Also Figure 1 shows fast observer error convergence to zero.

\section{CONCLUSION}

This paper introduces a new fault tolerant scheme for systems where only the system output information is available. Furthermore the scheme does not assume knowledge of the faults and failures from an FDI scheme. A linear unknown input observer is incorporated in the proposed FTC scheme to estimate the states, for use in the virtual control law. A control allocation scheme is also incorporated for distributing the virtual control effort. The closed-loop stability analysis allows some level of perturbation in the system matrix (due to airframe damage for example), and a convex representation of the synthesis problem is formulated. Simulation results on a benchmark aircraft model show good results.

\section{REFERENCES}

[1] H. Alwi and C. Edwards. Fault tolerant control using sliding modes with on-line control allocation. Automatica, (44):1859-1866, 2008.

[2] H. Alwi and C. Edwards. Fault tolerant sliding mode control design with piloted simulator evaluation. Journal of Guidance, Control, and Dynamics, 31(5):1186-1201, 2008.

[3] F.J. Bejarano, L. Fridman, and A. Poznyak. Output integral sliding mode control based on algebraic hierarchical observer. International Journal of Control, 80(3):443-453, 2007.

[4] J.D Bošković and R.K Mehra. Control allocation in overactuated aircraft under position and rate limiting. In $A C C, 2002$.

[5] J. Buffington. Tailless aircraft control allocation. In AIAA Guidance, Navigation and Control, pages 737-747, 1997.

[6] W. Cao and J. Xu. Nonlinear integral type sliding surface for both matched and unmatched uncertain systems. IEEE Transactions on Automatic Control, 49(8):1355-1360, 2004.

[7] F. Castanos and L. Fridman. Analysis and design of integral sliding manifolds for systems with unmatched perturbations. IEEE Transactions on Automatic Control, 51(5):853-858, 2006.

[8] J. Chang. Dynamic Output Integral Sliding-Mode Control With Disturbance Attenuation. IEEE Transactions on Automatic Control, 54(11):2653-2658, 2009.

[9] J.B. Davidson, F.J. Lallman, and W.T. Bundick. Real-time adaptive control allocation applied to a high performance aircraft. In 5th SIAM Conference on Control and Its Application, 2001.

[10] C. Edwards and S.K Spurgeon. Sliding Mode control, Theory and Applications. Taylor and Francis, 1998.

[11] C. Edwards, X. Yan, and S.K. Spurgeon. On the solvability of the constrained lyapunov problem. IEEE Transactions on Automatic Control, 52(9):1975-1981, 2007.

[12] M. Hamayun, C. Edwards, and H. Alwi. Integral Sliding Mode Fault Tolerant Control Incorporating On-line Control Allocation. In IEEE Workshop on Variable Structure Systems, pages 100-105, 2010.

[13] O. Harkegard and S.T Glad. Resolving actuator redundancy - optimal vs. control allocation. Automatica, (41):137-144, 2005.
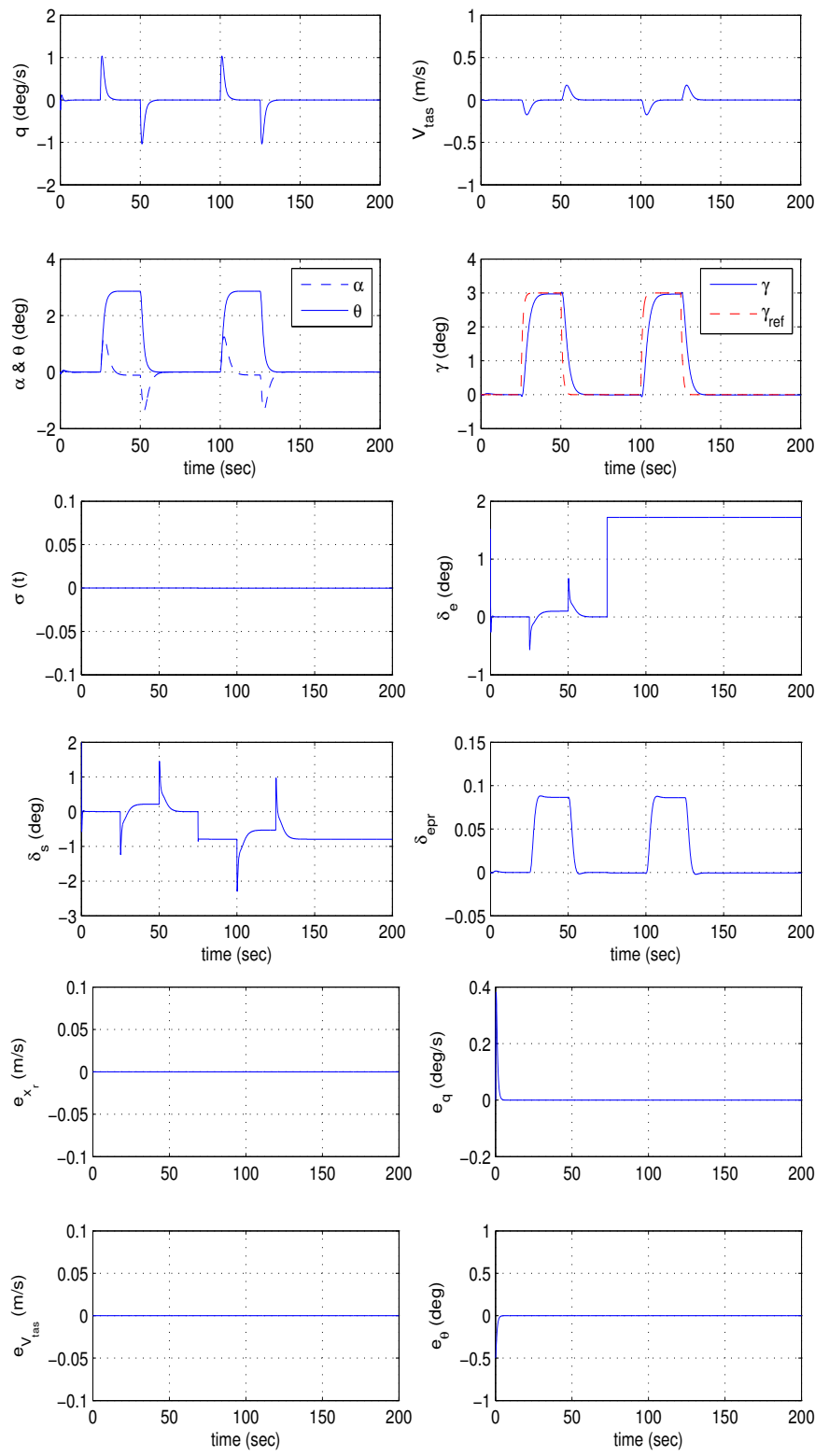

Fig. 1. Elevator jam: states, surface deflections and output error

[14] R.A. Hess and S.R. Wells. Sliding mode control applied to reconfigurable flight control design. Journal of Guidance, Control and Dynamics, (26):452-462, 2003.

[15] J.Chen and R. Patton. Robust model-based fault diagnosis for dynamical systems. Kluwer Acedemic Publshers, 1999.

[16] Y. Shtessel, J. Buffington, and S. Banda. Tailless aircraft flight control using multiple time scale re-configurable sliding modes. IEEE Transactions on Control Systems Technology, (10):288-296, 2002.

[17] G.W. Stewart. On scaled projections and pseudoinverses, volume 112 of Linear Algebra and its Applications. 1989.

[18] C.P. Tan and C. Edwards. Sliding mode observers for robust detection and reconstruction of actuator and sensor faults. International Journal of Roabust and nonlinear control, 13:443-463, 2003.

[19] V. Utkin, J. Guldner, and J. Shi. Sliding Mode control in Electrome chanical Systems. Taylor and Francis, 1999.

[20] V. Utkin and J. Shi. Integral sliding mode in systems operating under uncertainty conditions. In 35th IEEE CDC, 1996.

[21] M. Verhaegen, S. Kanev, R. Hallouzi, C. Jones, J. Maciejowski, and H. Smail. Fault tolerant flight control - a survey. In C. Edwards, T. Lombaerts, and H. Smaili, editors, Fault Tolerant Flight Control, volume 399 of LNCIS, pages 47-89. Springer, 2010. 